\title{
Human Melioidosis Caused by Novel Transmission of Burkholderia pseudomallei from Freshwater Home Aquarium, United States ${ }^{1}$
}

\author{
Patrick Dawson, ${ }^{2}$ Monique M. Duwell, ${ }^{2}$ Mindy G. Elrod, Ruth J. Thompson, David A. Crum, \\ Ruth M. Jacobs, Jay E. Gee, Cari B. Kolton, Lindy Liu, David D. Blaney, LaToya Griffin Thomas, \\ Denise Sockwell, Zachary Weiner, William A. Bower, Alex R. Hoffmaster, Johanna S. Salzer
}

\begin{abstract}
Nearly all cases of melioidosis in the continental United States are related to international travel to areas to which Burkholderia pseudomallei, the bacterium that causes melioidosis, is endemic. We report the diagnosis and clinical course of melioidosis in a patient from the United States who had no international travel history and the public health investigation to determine the source of exposure. We tested environmental samples collected from the patient's home for B. pseudomallei by PCR and culture. Whole-genome sequencing was conducted on PCR-positive environmental samples, and results were compared with sequences from the patient's clinical specimen. Three PCR-positive environmental samples, all collected from a freshwater home aquarium that had contained imported tropical fish, were a genetic match to the clinical isolate from the patient. This finding suggests a novel route of exposure and a potential for importation of B. pseudomallei, a select agent, into the United States from disease-endemic areas.
\end{abstract}

$\mathrm{M}$ elioidosis is a severe, potentially life-threatening bacterial disease caused by Burkholderia pseudomallei, a gram-negative bacterium found in water and soil in tropical and subtropical environments

Author affiliations: Centers for Disease Control and Prevention, Atlanta, Georgia, USA (P. Dawson, M.G. Elrod, J.E. Gee,

C.B. Kolton, L. Liu, D.D. Blaney, Z. Weiner, W.A. Bower,

A.R. Hoffmaster, J.S. Salzer); Maryland Department of Health, Baltimore, Maryland, USA (M.M. Duwell, R.J. Thompson,

D.A. Crum); Holy Cross Germantown Hospital, Germantown, Maryland, USA (R.M. Jacobs); Virginia Department of General Services, Richmond, Virginia, USA (L. Griffin Thomas); Virginia Department of Health, Richmond (D. Sockwell)

DOI: https://doi.org/10.3201/eid2712.211756 worldwide (1). Melioidosis might manifest as localized, pulmonary, systemic, or disseminated infections. However, melioidosis symptoms are nonspecific, and it is often misdiagnosed $(1,2)$. Exposure to $B$. pseudomallei occurs through inhalation of contaminated dust or water droplets, ingestion of contaminated water, and direct contact with contaminated water or soil, particularly through cuts or abrasions (3). The incubation period in acute cases ranges from 1 to 21 days, although activation of latent infections might develop many years later $(4,5)$. Persons at greater risk for developing melioidosis include those with diabetes, liver disease, renal disease, chronic lung disease, thalassemia, cancer, and other immunocompromising conditions $(6,7)$.

Melioidosis (formerly Whitmore's disease) was described in 1912 (8), and cases were historically identified primarily in northern Australia and areas of Southeast Asia, such as Thailand, where melioidosis is hyperendemic (9). However, the known geographic range has expanded considerably in recent decades; the estimated global burden is $165,000 \mathrm{hu}-$ man cases/year (10). On the basis of clinical cases or environmental isolation, $B$. pseudomallei is now suspected to be endemic to the environment in parts of Central and South America, the Caribbean, Mexico, and potentially in areas of the continental United States, such as Texas (11-14). Despite increased recognition of the expansive range of B. pseudomallei,

${ }^{1}$ Preliminary results of this study were presented at the Virtual IDWeek 2020 Conference; October 21-25, 2020.

${ }^{2}$ These authors contributed equally to this article. 
nearly all US cases are related to previous residence in or travel to disease-endemic areas outside the continental United States (15).

\section{Case Report}

In October 2019, the US Centers for Disease Control and Prevention (CDC) was notified by the Maryland Department of Health and through the CDC Laboratory Response Network (LRN) of a preliminary positive bacterial isolate of $B$. pseudomallei from a blood culture specimen from a Maryland resident. The clinical specimen had been forwarded to the Virginia Division of Consolidated Laboratory Services, where it was confirmed, and shipped to CDC for simultaneous confirmation and genomic analysis.

The patient, a 56-year-old woman, was hospitalized on September 20, 2019 (day 0; Figure), because of fever, cough, and chest pain with onset 2 days earlier. The patient had a history of polymyositis, rheumatoid arthritis, and diabetes mellitus, and reported to have stopped long-term immunosuppressive medications (methotrexate, azathioprine, and prednisone) 1 month before symptom onset.

A thoracic radiograph on day 0 showed a right perihilar and lower lobe infiltrate, consistent with

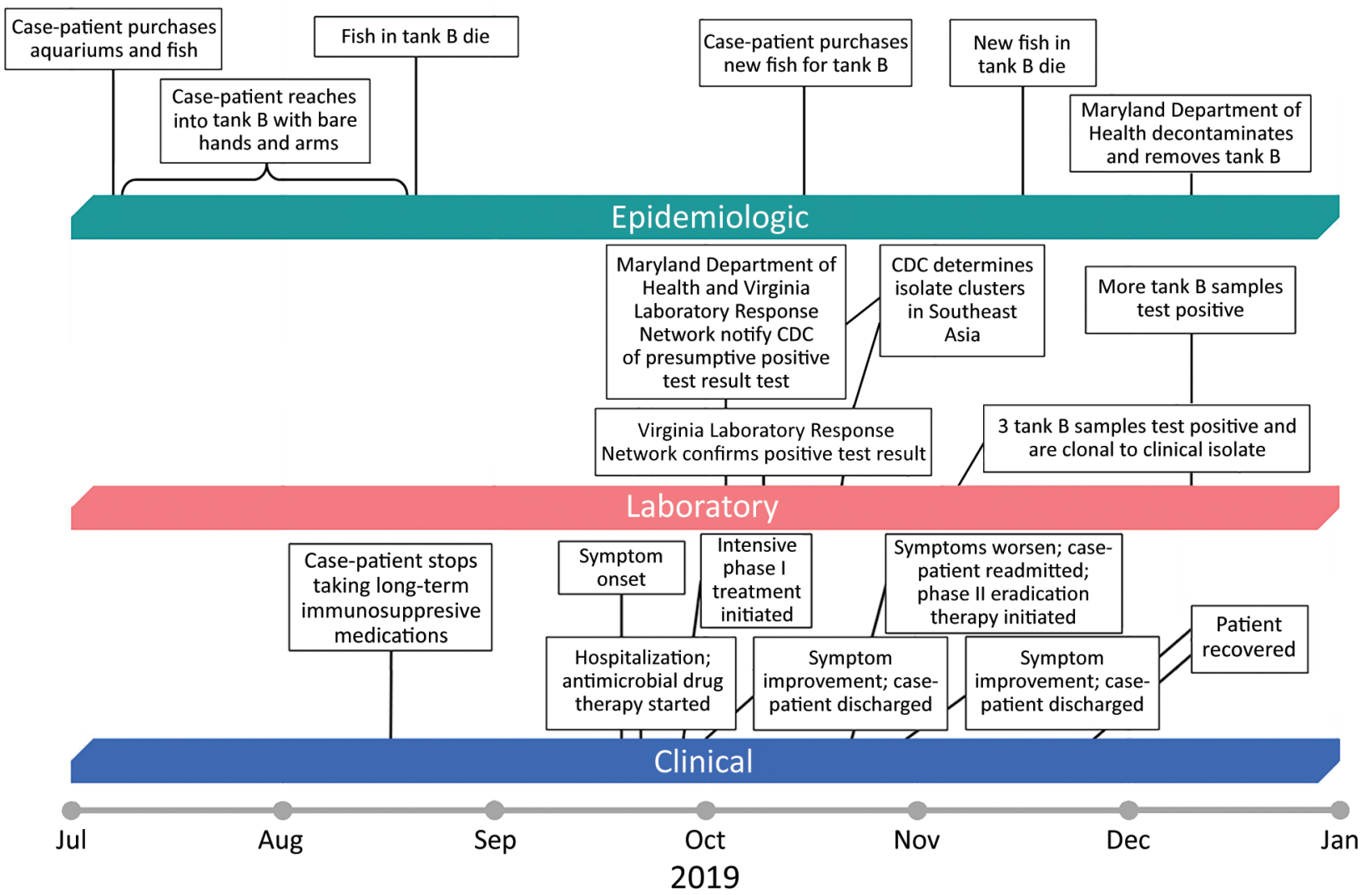

Figure. Clinical, laboratory, and epidemiologic timeline for a patient who had melioidosis, Maryland, USA, 2019. CDC, Centers for Disease Control and Prevention. pneumonia. A noncontrast computed tomography (CT) scan on day 3 showed air space consolidation in the right lower lobe consistent with pneumonia, additional bibasilar air space densities, and a small right pleural effusion. Other notable clinical laboratory results at presentation included an increased leukocyte count of 22,800 cells/ $\mu \mathrm{L}$ (reference range 4,500-11,000 cells $/ \mu \mathrm{L}$ ) and a decreased sodium level of $125 \mathrm{mmol} / \mathrm{L}$ (reference range 135-145 mmol/L).

We obtained blood cultures on day 0 before administration of antimicrobial drugs. Gram-negative rods were identified in 4 initial blood cultures; the rods were subsequently identified as B. pseudomallei. Three additional blood cultures obtained on days 2-4 grew the same organism. The patient was given ceftriaxone and azithromycin on days $0-3$, then on day 4 , treatment was changed to meropenem after B. pseudomallei was identified. The patient showed gradual symptom improvement; fever resolved, and leukocyte count normalized. The intensive phase duration was extended because of persistent bacteremia. She was discharged on day 11 and continued taking intravenous meropenem as an outpatient. Antimicrobial drug susceptibility testing was not performed because the patient had been

\section{Epidemiologic}

Health and Virginia
Laboratory Response
Network notify CDC
of presumptive positive
test result test

Virginia Laboratory Response

positive test result

sitive and

\section{are clonal to clinical isolate} an 
responding to treatment with meropenem before LRN confirmation of $B$. pseudomallei.

After 3 weeks of taking meropenem, the patient's leukocyte count increased to 14,700 cells $/ \mu \mathrm{L}$, and fever developed (temperature $100.1^{\circ} \mathrm{F}\left[37.8^{\circ} \mathrm{C}\right]$ ). A repeat CT scan showed a patchy opacity at the right lung base and bilateral interstitial process consistent with pneumonia, but a $45 \times 24 \mathrm{~mm}$ mass in the right lung posteriorly could not be excluded and increased concern for pulmonary abscess. The patient was readmitted, sulfamethoxazole/trimethoprim was given, and treatment with meropenem continued. She clinically improved and was discharged after 1 week. Repeat, noncontrast, thoracic CT scans showed improvement of right basilar consolidation after 6 weeks of meropenem and sulfamethoxazole/ trimethoprim and resolution after 10 weeks. The patient completed eradication therapy with a 10week course of meropenem and 12-week course of sulfamethoxazole/trimethoprim.

\section{Methods}

\section{Case Investigation}

We conducted an initial epidemiologic investigation to assess the travel history of the patient and other possible exposures to $B$. pseudomallei. Interviews were conducted October-December 2019, with the patient and other household members. After establishing no international travel history and identification of positive environmental samples, the interviews focused on the freshwater home aquariums, tropical fish, and contact with the fish and aquariums of the patient.

\section{Environmental Sampling}

During November 2019, the investigation team visited the home of the patient to collect environmental samples and assess potential sources of B. pseudomallei. The patient had 2 freshwater aquariums (tanks A and $B$, both $\approx 5$ liters). We collected bulk water samples $(\approx 50 \mathrm{~mL})$ from each aquarium and swab specimens of biofilms ( 3 from tank A and 2 from tank B). We collected an additional 16 samples, including swab specimens of all household faucets, soil from potted plants and around the property, 2 beauty products, and 4 liquid vaping products. During December 2019, the team collected 6 additional specimens: bulk water from tanks $A$ and $B$, gravel from tank B, filters from tank $B$, dead fish carcasses from tank $B$, and a dry artificial plant that had been in tank B but was removed during August 2019. During the second visit, the Maryland Department of Health also performed decontamination, removal, and safe disposal of fish tank B from the home of the patient.

\section{Laboratory Confirmation}

The clinical isolate was confirmed as B. pseudomallei at the Virginia Division of Consolidated Laboratory Services and CDC by using the LRN algorithm, including biochemicals and PCR. All 29 environmental samples were sent to CDC for culture and identification. We directly inoculated all environmental samples into TBSS-C50 (Galimand) broth and incubated broths in a shaking incubator at $37^{\circ} \mathrm{C}$ for 6 days. We then cultured enriched broths onto Ashdown agar, and extracted DNA by using a QIAamp Fast Stool Mini Kit (QIAGEN, https://www.qiagen.com) or testing by real-time PCR. Suspected colonies from Ashdown agar were selected for further workup, and confirmation of isolates followed the LRN algorithm.

\section{Multilocus Sequence Typing and Whole-Genome Sequencing}

CDC performed multilocus sequence typing on isolate MD2019a (clinical). CDC also performed wholegenome sequencing (WGS) for isolates MD2019a (clinical), MD2019b (water from tank B collected during November 2019), and MD2019c (swab specimen of biofilms from tank B collected during November 2019) by using the Nextera FLEX Kit (https:/ / www. illumina.com) for library preparation and the iSeq 100 instrument (Illumina) with a $2 \times 151$-bp kit. We analyzed draft genomes along with a reference panel of $B$. pseudomallei genomes from publicly available sources representing geographic diversity by using Parsnp in the HARVEST 1.3 suite (https://harvest. readthedocs.io/en/latest/content/parsnp.html) to detect single-nucleotide polymorphisms (SNPs).

\section{Results}

\section{Initial Investigation}

The initial epidemiologic investigation showed that the patient was a long-time resident of Maryland who had never traveled outside the continental United States. She reported previous pet ownership of reptiles and cats $>5$ years ago. She denied any other direct soil or environmental water exposure. She also denied use of herbal products or products known to be imported from Asia or Australia. She reported use of vaping products. No family members or close contacts had an illness similar to that of the patient.

Multilocus sequence typing yielded sequence type 369, which had been seen in examples from Malaysia, Thailand, and Vietnam (16). WGS of the 
clinical isolate, MD2019a, showed that when compared to a panel of publicly available genomes composed to represent geographic diversity, it clustered with genomes from Southeast Asia predominantly associated with Singapore and Malaysia (Appendix Figure, https://wwwnc.cdc.gov/EID/ article/27/12/21-1756-App1.pdf). The genetic link of the isolate to Southeast Asia, coupled with lack of international travel history for the patient, led the investigation team to conduct follow-up interviews and environmental sampling in the home of the patient.

\section{Isolation of $B$. pseudomallei from Environmental Samples}

Of the 23 environmental samples collected during November 2019, a total of 3 samples, all from tank B, were positive for $B$. pseudomallei by culture and realtime PCR. All other November 2019 samples were negative. The water and gravel samples from tank B collected during December 2019 were also positive for B. pseudomallei by PCR and culture. The artificial plant that was removed from the tank during August 2019 was positive by PCR, but no growth was observed in culture. The December 2019 water sample from tank A was negative.

\section{Genetic Match of Aquarium Isolates with the Clinical Isolate}

Comparison of the draft genome sequences between the clinical isolate (MD2019a) and the 2 aquarium samples from tank B (MD2019b and MD2019c) showed no SNPs between MD2019a and MD2019c, and only 1 SNP was detected between MD2019a and MD2019b. This finding indicates that all 3 isolates were clonal.

\section{Follow-Up Patient Interviews}

The patient reported she had purchased fish, aquariums, and associated supplies during July 2019 (Figure). She purchased the tanks and gravel substrate at a large retail store. She purchased 3 cherry barbs (Puntius titteya) for tank A from a retail pet store, and all were alive at the time of her interview. She purchased 3 fancy-tailed guppies (Poecilia reticulata) for tank B from the same store, and they died during August 2019. During October 2019, after her illness onset, she purchased 3 tiger barbs (Puntigrus tetrazona) for tank B from the same store, and they died during November 2019.

The patient reported that the water in tank B was persistently cloudier than the water in tank A and more difficult to keep clean. She reported that she was the primary caretaker of the fish and recalled reaching bare hands and arms into the water and gravel of tank B to disrupt sediment while cleaning it, as recently as August 2019.

\section{Discussion}

This investigation of a patient who had melioidosis and no history of travel to disease-endemic areas provides strong evidence for documented transmission of B. pseudomallei from a freshwater home aquarium to a human. Although this type of transmission has not been described in the literature, contamination of aquarium transport water with B. pseudomallei used for freshwater tropical fish originating from Singapore has been reported in France (17). Furthermore, freshwater fishing practices have been identified as risk factors in countries to which melioidosis is endemic $(7,18,19)$.

With freshwater aquariums as a newly recognized source of possible transmission of $B$. pseudomallei to humans, further investigations are underway to determine the extent of $B$. pseudomallei contamination at the pet store retailer where the patient purchased the pet fish with accompanying aquarium water and at the vendors that imported and supplied freshwater ornamental fish, aquatic plants, and associated aquarium water to this retail location. Because these vendors might distribute freshwater animals and aquatic plants to pet store retailers throughout the United States, identifying possible source(s) of introduction with B. pseudomallei in the supply chain is essential to public health.

Federal regulators classify $B$. pseudomallei as a Tier 1 Select Agent because of its heightened risk for deliberate misuse and major potential for mass casualties, economic disruption, critical infrastructure effects, or damaging public confidence (20). The United States is the largest importer of ornamental fish, most of which are freshwater and originate in Southeast Asia $(21,22)$, where B. pseudomallei is widespread in the environment. An estimated 11.5 million US households have pet fish; $\approx 139$ million freshwater fish are owned $(23,24)$. Determining where in the supply chain introduction of the bacteria might occur can lead to development of enhanced surveillance and mitigation procedures at the critical control points, which might prevent further introductions and spread of the bacteria to retailers and homes of consumers.

To prevent or reduce risk of exposure, particularly among persons who have major risk factors, simple precautions can be taken when handling freshwater fish, snails, aquatic plants, aquariums, or other materials in contact with aquarium 
water, such as gravel, substrate, decorations, filters, and other equipment. CDC recommends thorough handwashing with soap and water before and after handling or cleaning aquariums and feeding fish, wearing gloves to cover any cuts or wounds in the hand while handling fish or aquariums or allowing wounds to fully heal first, avoiding cleaning fish aquariums if immunocompromised or in areas where immunocompromised persons might be present, and not allowing children $<5$ years of age to clean fish aquariums (25).

This report highlights the essential role of molecular epidemiology in public health investigations of melioidosis cases, which identified the likely geographic origin of the bacteria and prompted a public health response that characterized a novel route of exposure. There is growing evidence that US melioidosis cases are not limited to international travelers, including a 2021 multistate cluster involving an organism that is not clonal to the isolates described here, as determined by WGS (14,26-29).

We urge clinicians in the United States to consider melioidosis in patients who have clinically compatible symptoms and exposure to tropical ornamental fish and freshwater aquariums, particularly if patients are immunocompromised, even though such exposure events might be exceedingly rare and few persons show development of melioidosis after exposure to B. pseudomallei (5). This organism might be difficult for hospital laboratories to diagnose, and automated identification systems in clinical laboratories can misidentify B. pseudomallei, highlighting the need for LRN confirmation (28).

Clinicians treating melioidosis should consult established treatment guidelines, which were updated in $2020(30,31)$. Likewise, public health investigators should consider inquiring about pet freshwater fish exposure in patients given a diagnosis of melioidosis who have not traveled to a disease-endemic area or have only traveled to locations inconsistent with the geographic profile of the genome of their isolate.

\section{Acknowledgment}

We thank David Blythe for providing contributions to this study.

\section{About the Author}

Dr. Dawson is an epidemiologist in the Office of Science, Centers for Disease Control and Prevention, Atlanta, GA. His primary research interests are emerging infectious diseases, zoonotic diseases, and health equity.

\section{References}

1. Wiersinga WJ, Currie BJ, Peacock SJ. Melioidosis. N Engl J Med. 2012;367:1035-44. https:/ / doi.org/10.1056/ NEJMra1204699

2. Hoffmaster AR, AuCoin D, Baccam P, Baggett HC, Baird R, Bhengsri S, et al. Melioidosis diagnostic workshop, 2013. Emerg Infect Dis. 2015;21:e141045.

3. Centers for Disease Control and Prevention. Melioidosis transmission [cited 2021 Sep 17]. https:/ / www.cdc.gov/ melioidosis/transmission/index.html.

4. Currie BJ, Fisher DA, Howard DM, Burrow JN, Selvanayagam S, Snelling PL, et al. The epidemiology of melioidosis in Australia and Papua New Guinea. Acta Trop. 2000;74:121-7. https://doi.org/10.1016/ S0001-706X(99)00060-1

5. Currie BJ. Melioidosis: evolving concepts in epidemiology, pathogenesis, and treatment. Semin Respir Crit Care Med. 2015;36:111-25. https:// doi.org/10.1055/s-0034-1398389

6. Centers for Disease Control and Prevention. Melioidosis signs and symptoms [cited 2021 Sep 17]. https:/ / www.cdc. gov/melioidosis/symptoms/index.html

7. Currie BJ, Ward L, Cheng AC. The epidemiology and clinical spectrum of melioidosis: 540 cases from the 20 year Darwin prospective study. PLoS Negl Trop Dis. 2010;4:e900. https:// doi.org/10.1371/journal.pntd.0000900

8. Whitmore A, Krishnaswami CS. A hitherto undescribed infective disease in Rangoon. Ind Med Gaz. 1912;47:262-7.

9. Currie BJ, Dance DA, Cheng AC. The global distribution of Burkholderia pseudomallei and melioidosis: an update. Trans R Soc Trop Med Hyg. 2008;102(Suppl 1):S1-4. https:/ / doi.org/10.1016/S0035-9203(08)70002-6

10. Limmathurotsakul D, Golding N, Dance D, Messina J, Pigott D, Moyes C, et al. Predicted global distribution of Burkholderia pseudomallei and burden of melioidosis. Nat Microbiol. 2016;1:15008. https:/ / doi.org/10.1038/ nmicrobiol.2015.8

11. Benoit TJ, Blaney DD, Doker TJ, Gee JE, Elrod MG, Rolim DB, et al. A review of melioidosis cases in the Americas. Am J Trop Med Hyg. 2015;93:1134-9. https:/ / doi.org/10.4269/ajtmh.15-0405

12. Sanchez-Villamil JI, Torres AG. Melioidosis in Mexico, Central America, and the Caribbean. Trop Med Infect Dis. 2018;3:24. https://doi.org/10.3390/tropicalmed3010024

13. Doker TJ, Sharp TM, Rivera-Garcia B, Perez-Padilla J, Benoit TJ, Ellis EM, et al. Contact investigation of melioidosis cases reveals regional endemicity in Puerto Rico. Clin Infect Dis. 2015;60:243-50. https:/ / doi.org/10.1093/ cid/ciu764

14. Cossaboom CM, Marinova-Petkova A, Strysko J, Rodriguez G, Maness T, Ocampo J, et al. Melioidosis in a resident of Texas with no recent travel history, United States. Emerg Infect Dis. 2020;26:1295-9. https:/ / doi.org/10.3201/ eid2606.190975

15. Elrod MG, Gee JE, Gulvik CA, Liu L, Salzer JS, Deka MA, et al. Update on melioidosis in the Americas. Presented at: 9th World Melioidosis Congress; Hanoi, Vietnam; October 15-18, 2019.

16. Godoy D, Randle G, Simpson AJ, Aanensen DM, Pitt TL, Kinoshita R, et al. Multilocus sequence typing and evolutionary relationships among the causative agents of melioidosis and glanders, Burkholderia pseudomallei and Burkholderia mallei. J Clin Microbiol. 2003;41:2068-79. https://doi.org/10.1128/JCM.41.5.2068-2079.2003

17. Galimand M, Escallier G, Dodin A. The health risks of importing tropical fish [in French]. Revue Française d'Aquariologie Herpétologie. 1981;8:19-22. 
18. Hassan MR, Pani SP, Peng NP, Voralu K, Vijayalakshmi N, Mehanderkar R, et al. Incidence, risk factors and clinical epidemiology of melioidosis: a complex socio-ecological emerging infectious disease in the Alor Setar region of Kedah, Malaysia. BMC Infect Dis. 2010;10:302. https://doi.org/10.1186/1471-2334-10-302

19. Fang Y, Chen H, Li Y-L, Li Q, Ye Z-J, Mao X-H. Melioidosis in Hainan, China: a restrospective study. Trans R Soc Trop Med Hyg. 2015;109:636-42. https://doi.org/10.1093/trstmh/ trv065

20. Federal Select Agent Program. Biosafety/biocontainment plan guidance [cited 2021 Sep 17]. https:/ / www.selectagents. gov/bbp-definitions.html

21. Dey $\mathrm{V}$. The global trade in ornamental fish. Infofish International. 2016;4:52-5.

22. Livengood EJ, Chapman FA. The ornamental fish trade: an introduction with perspectives for responsible aquarium fish ownership. Gainesville (FL): US Department of Agriculture, University of Florida, UF/IFAS Extension Service; 2007.

23. American Pet Products Association. 2019-2020 APPA national pet owners survey, 2020 [cited 2021 Sep 17]. https:/ / www.americanpetproducts.org/press_industrytrends.asp

24. American Pet Products Association. 2017-2018 APPA national pet owners survey, 2018 [cited 2021 Sep 17]. https://www.americanpetproducts.org/press_industrytrends.asp.

25. Centers for Disease Control and Prevention. Healthy pets, healthy people: fish [cited 2021 Sep 17]. https:/ / www.cdc. gov/healthypets/pets/fish.html

26. Hall CM, Jaramillo S, Jimenez R, Stone NE, Centner H, Busch JD, et al. Burkholderia pseudomallei, the causative agent of melioidosis, is rare but ecologically established and widely dispersed in the environment in Puerto Rico. PLoS Negl Trop Dis. 2019;13:e0007727. https://doi.org/10.1371/ journal.pntd.0007727

27. Stewart T, Engelthaler DM, Blaney DD, Tuanyok A, Wangsness E, Smith TL, et al. Epidemiology and investigation of melioidosis, Southern Arizona. Emerg Infect Dis. 2011;17:1286-8. https://doi.org/10.3201/eid1707.100661

28. Doker TJ, Quinn CL, Salehi ED, Sherwood JJ, Benoit TJ, Glass Elrod M, et al.; Melioidosis Investigation Team. Fatal Burkholderia pseudomallei infection initially reported as a Bacillus species, Ohio, 2013. Am J Trop Med Hyg. 2014;91:743-6. https://doi.org/10.4269/ajtmh.14-0172

29. Centers for Disease Control and Prevention. CDCHAN-00448. New case identified: multistate investigation of non-travel associated Burkholderia pseudomallei infections (melioidosis) in four patients: Georgia, Kansas, Minnesota, and Texas-2021, August 9, 2021 [cited 2021 Sep 9]. https://emergency.cdc.gov/han/2021/han00448.asp

30. Pitman MC, Luck T, Marshall CS, Anstey NM, Ward L, Currie BJ. Intravenous therapy duration and outcomes in melioidosis: a new treatment paradigm. PLoS Negl Trop Dis. 2015;9:e0003586. https://doi.org/10.1371/ journal.pntd.0003586

31. Sullivan RP, Marshall CS, Anstey NM, Ward L, Currie BJ. 2020 Review and revision of the 2015 Darwin melioidosis treatment guideline; paradigm drift not shift. PLoS Negl Trop Dis. 2020;14:e0008659. https:/ / doi.org/10.1371/ journal.pntd.0008659

Address for correspondence: Patrick Dawson, Centers for Disease Control and Prevention, 1600 Clifton Rd NE, Mailstop H21-8, Atlanta, GA 30329-4027, USA; email: wpb7@cdc.gov 